\title{
Cellulose Nanostructures Obtained from Waste Paper Industry: A Comparison of Acid and Mechanical Isolation Methods
}

\author{
Alana Gabrieli de Souza ${ }^{a}$, Fabiany Sayuri Kano ${ }^{a}$, Jean Jacques Bonvent ${ }^{a}$, Derval dos Santos Rosa ${ }^{a}$ \\ ${ }^{a}$ Universidade Federal do ABC (UFABC), Santo André, SP, Brazil
}

Received: November 20, 2016; Revised: April 19, 2017; Accepted: June 03, 2017

\begin{abstract}
The paper industry primary waste is usually deposited in landfills or incinerated. This material has a high content of lignocellulosic components, which could be converted to cellulose nanostructures (CNS). This study aimed to compare compositional (Fourier transform infrared spectroscopy), thermal (thermogravimetric analysis), morphological (scanning electron microscopy) and dimensional (dynamic light scattering and atomic force microscopy) properties of CNS obtained through chemical and mechanical isolation processes. The FTIR results showed similar chemical bonding for both nanostructures, which indicated presence of cellulose and lignin. Their average size was $170 \mathrm{~nm}$ and $209 \mathrm{~nm}$ for chemical and mechanical processes, respectively. The morphology of SEM images showed a compact structure and the chemical isolation presents smaller CNS agglomeration than mechanical. TGA results showed higher thermal stability for CNS-chemical than CNS-mechanical samples and AFM images indicate the CNS morphologies, which showed nanoroads for CNS-chemical and nanoneedles for CNS-mechanical.
\end{abstract}

Keywords: Nanostructure, nanocellulose, paper waste, acid hydrolysis, ball milling

\section{Introduction}

The growing awareness of environmental issues and resource scarcity explains the increased interest by industries in the reuse of wastes generated during production processes. Furthermore, strict legislative policies have forced the industries to find ways to reuse residues that previously were incinerated or disposed in landfills ${ }^{1}$.

The paper industry is considered one of the largest consumers of natural resources (wood and water) and energy (electricity and fossil fuels). This kind of industry also contributes significantly to emissions of pollutants to the environment. These pollutants are the result of chemical treatment carried out in the pulping of wood and paper production. The final sludge resulting from the production process consists of $25-30 \%$ lignocellulosic material and $70-75 \%$ water $^{2}$.

The conversion of lignocellulosic materials in nanocellulose has been the focus of recent research aimed at the reuse and revalorization of waste. Industrial wastes such as tobacco and cotton industry waste ${ }^{3,4}$ have been studied as sources of cellulose nanostructures (CNS). The isolation of CNS has attracted significant interest for a broad range of applications, such as nanocomposites, due to their nontoxicity, biodegradability, low density, excellent mechanical properties and nanosize dimension with higher surface area, hence, a huge interface with the matrix polymers ${ }^{5}$. The usual isolation methods to obtain nanocelluloses are chemical and mechanical. Chemical

*e-mail: dervalrosa@yahoo.com.br isolation by acid hydrolysis results in a disorder of molecules that destroys amorphous cellulose, resulting in crystalline nanocellulose. For the mechanical process, the mechanism of fibrillation in grinder is to break down of hydrogen bonds and cell wall structure by shear forces and individualization of pulp to nanoscale fibers ${ }^{6}$.

In this study, CNS were prepared from primary residues of the paper industry as an alternative to minimize the volume of incinerated materials and to add value to this lignocellulosic material. The composition of this paper waste includes lignocellulosic components, sodium hydroxide, sodium carbonate, sodium sulfite, calcium oxide and others ${ }^{7}$. Two methodologies were used for isolation: chemical, which used sulfuric acid, and mechanical, which used ball mill. Thermal, morphological, and dimensional properties were evaluated to determine which method was more effective, with higher thermal stability and smaller particle sizes.

\section{Experimental}

Materials: The raw material is primary paper residue provided by Multiverde Papeis Especiais Ltda. Company (Mogi das Cruzes, Brazil).

Treatments: The CNSs were obtained through two pretreatments: mercerization followed by bleaching. The mercerization was done mixing $1 \mathrm{~g}$ of residue and $20 \mathrm{~mL}$ of $92 \%(\mathrm{v} / \mathrm{v})$ acetic acid and $1.4 \%(\mathrm{v} / \mathrm{v})$ sulfuric acid aqueous solution with constant agitation at $115^{\circ} \mathrm{C}$ for $3 \mathrm{~h}$. Later, the samples were washed by distilled water, filtered and dried at $50{ }^{\circ} \mathrm{C}$ for $3 \mathrm{~h}$. Bleaching was done submitting the mercerized 
samples in $34 \%(\mathrm{v} / \mathrm{v})$ hydrogen peroxide and $4 \%(\mathrm{w} / \mathrm{v})$ sodium hydroxide aqueous solution, with constant agitation at $50{ }^{\circ} \mathrm{C}$ for $1 \mathrm{~h}$. Then the samples were washed, filtered and dried at $80^{\circ} \mathrm{C}$ for $2 \mathrm{~h}$. The CNS isolation was conducted by two different methodologies: (I) Acid hydrolysis was done with $40 \%$ sulfuric acid aqueous solution $\left(\mathrm{H}_{2} \mathrm{SO}_{4}\right)$ at $50{ }^{\circ} \mathrm{C}$ for 1 h. The solution was centrifuged at $6000 \mathrm{RPM}$ for $10 \mathrm{~min}$ and neutralized in a dialysis membrane for five days, and (II) Mechanical grinding by ball milling for $4 \mathrm{~h}$, using $1 \mathrm{~g}$ of residue and $80 \mathrm{~g}$ of porcelain balls with $21 \mathrm{~mm}$ diameter.

\subsection{Characterizations:}

(i) Fourier transform infrared spectroscopy (FTIR): FTIR analysis was used to identify the chemical structure of the lignocellulosic components present in the samples. The equipment was a Frontier 94942 (PerkinElmer, USA). The resolution of this instrument is $2 \mathrm{~cm}^{-1}$. Using attenuated total reflectance accessory, the spectra were recorded in the range of $400-4000 \mathrm{~cm}^{-1}$, and 64 scans were carried out.

(ii) Thermogravimetric analysis (TGA): Thermal stability was evaluated by progressive increasing of the temperature increases in the thermogravimetric analyzer (STA 6000, PerkinElmer, USA). About $5 \mathrm{mg}$ of samples were placed in alumina pan and heated from 20 to $600{ }^{\circ} \mathrm{C}$ at the rate of $20{ }^{\circ} \mathrm{C} \mathrm{min}^{-1}$. The flow of dynamic carrier nitrogen gas was set at a rate of $50 \mathrm{~mL} \mathrm{m^{-1 }}$.

(iii) Scanning electron microscopy (SEM): The surface morphology was analyzed by SEM (JEOL - JCM 600, 20 $\mathrm{kV})$. The CNS suspensions were dried at $50^{\circ} \mathrm{C}$ for $3 \mathrm{~h}$ and the samples were covered with gold by scattering equipment (SCANCOAT, PIRANI 501) at a pressure of $0.3 \mathrm{mbar}$ at $1.5 \mathrm{kV}$ for $35 \mathrm{~s}$.

(iv) Dynamic light scattering (DLS): The hydrodynamic radius was obtained as an approximated crystal size of the nanocellulose dispersed in water. The information was obtained using a dynamic light scattering system (ALV-CGS3) with $90^{\circ}$ fixed scattering angle. A HeNe polarized laser $(22 \mathrm{~mW})$ with a wavelength of $633 \mathrm{~nm}$ was used.

(v) Atomic force microscopy (AFM): The surface morphology and topography of the CNS-chemical and CNS-mechanical samples were analyzed by AFM. For images examination, a droplet of the aqueous suspension was sprayed onto a freshly cleaned silicon wafer and then airdried during $24 \mathrm{~h}$, at room temperature. The AFM experiments were performed in tapping mode, using an Agilent equipment (model 5500), at ambient relative humidity and temperature. The scan was done at a rate of $0.5 \mathrm{~Hz}$, with an image resolution of $256 \times 256$ pixel, using a silicon cantilever (nanosensor) of constant force between 25 and $75 \mathrm{~N} \mathrm{~m}-1$ and resonance frequency of 120 $\mathrm{kHz}$. The topography, phase and amplitude of the images were simultaneous recorded for further analysis.

\section{Results and Discussion}

(i) Fourier transform infrared spectroscopy

Figure 1 shows the FTIR spectra of the paper residue, CNS-chemical and CNS-mechanical samples. It demonstrates the changes in chemical composition of the fibers. The variations relative to the conversion from macro- to nanomaterials are monitored by the changes in the hydroxyl and carboxyl regions. The peaks 3340 and $2918 \mathrm{~cm}^{-1}$, present in all the samples, were due to hydroxyl group and to aliphatic saturated C-H stretching vibrations of cellulose, respectively ${ }^{8}$. The peak at $1640 \mathrm{~cm}^{-1}$ associated with the $\mathrm{O}-\mathrm{H}$ bending vibration of absorbed water was evident in spectra of all samples ${ }^{9,10}$. With regard to the possible effects of extractions on the transmittance bands, it was observed smoothed peaks in the region between 1100 and $1500 \mathrm{~cm}^{-1}$, where it is not possible to attributed signatures for particular vibrations, since, in this region, complex overlap effects may occur ${ }^{3}$. These small peaks could be also from hemicelluloses and proteins which are present in the cellulose fiber walls ${ }^{11,12}$. The spectra of all samples are typical of cellulose. Presenting peaks at 1060 and $896 \mathrm{~cm}^{-1}$ which indicates, according the literature, the purity of the crystalline band of cellulose, with characteristics of $\mathrm{C}-\mathrm{O}$ stretching vibration and elongation of cellulose typical pulp $\beta$-glycoside bonds, respectively, especially in nanocellulose spectra (CNS-chemical and CNSmechanical $)^{3,9-13}$. It is important to note that the extracted nanocellulose shows transmittance signal at $1428,1160 \mathrm{~cm}^{-1}$ (indicated by arrow in Figure 1) and $896 \mathrm{~cm}^{-1}$ which show that nanocellulose produced was primarily in the form of cellulose I structure, which is the form of native cellulose $\mathrm{e}^{14,15}$.

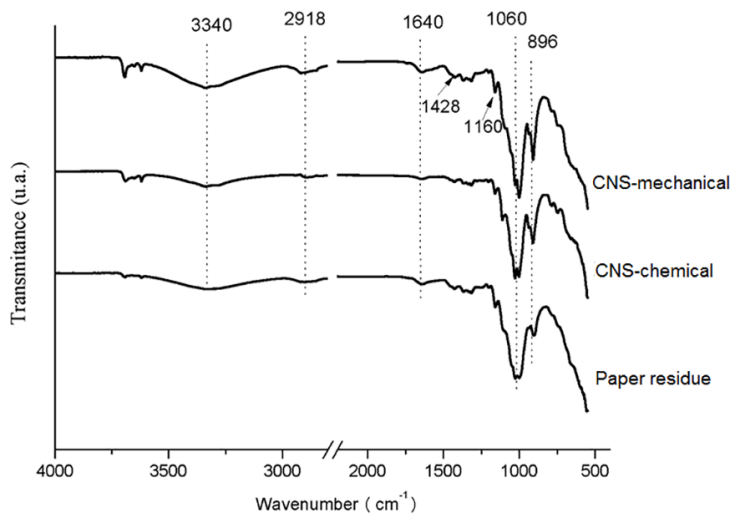

Figure 1. FT-IR spectrum of the paper residue, CNS-chemical and CNS-mechanical samples.

(ii) Thermogravimetric analysis (TGA)

Figure 2 shows the thermal profile of the all samples. The first step of thermal degradation (Figure 2(a)) was observed around $100^{\circ} \mathrm{C}$, which was mainly associated to the evaporation of water due to the hydrophilic character of the fibers ${ }^{9,10}$. 

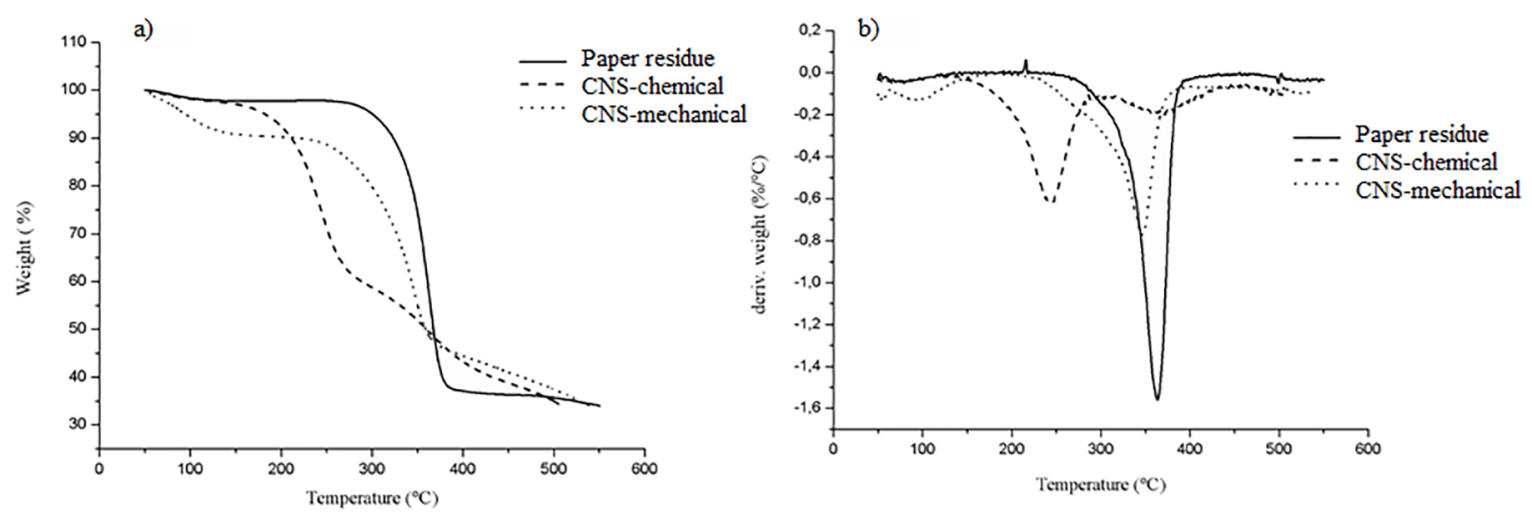

Figure 2. (a) Thermal curves (TG) and; (b) derivative curves (DTG) obtained by TGA for the paper residue, CNS-chemical and CNSmechanical samples.

The presence of absorbed water was also detected by FTIR characteristic peak at $1640 \mathrm{~cm}^{-1}$. The initial decomposition temperature (measured at $10 \%$ weight loss) for the paper residue, CNS-chemical and CNS-mechanical were $310^{\circ} \mathrm{C}$, $196^{\circ} \mathrm{C}$, and $140^{\circ} \mathrm{C}$, respectively. The paper residue showed a higher initial thermal degradation than CNS-chemical and CNS-mechanical samples due to the presence of the fiber constituents (lignin and hemicellulose). These components serve as barriers to initiate its degradation ${ }^{16}$. Around 170 ${ }^{\circ} \mathrm{C}$, the thermal degradation is associated with the release of volatile and non-polymeric compounds, which can be observed to CNS-chemical and CNS-mechanical samples ${ }^{3}$. CNS showed lower thermal stability than the paper residue, which could be attributed to an increase in the surface area of nanoparticles ${ }^{9,17,18}$.

The DTG curves (Figure 2(b)) revealed the temperature for maximum rate of weight loss for all samples, which are $364{ }^{\circ} \mathrm{C}$ for the paper residue, $243{ }^{\circ} \mathrm{C}$ and $371{ }^{\circ} \mathrm{C}$ for the CNS-chemical and $345^{\circ} \mathrm{C}$ for CNS-mechanical. The CNSchemical showed two different degradations peaks, indicating the presence of different components that decompose at different temperatures. The first may be associated with the sulfate groups on the surface, which induces a higher thermal degradation rate. The second peak, present in all samples, it is around $300{ }^{\circ} \mathrm{C}$ and represents the maximum rate of cellulose degradation ${ }^{3}$. The CNS-chemical sample present higher $\mathrm{T}_{\text {max }}$ and initial decomposition temperature than CNS-mechanical sample. That might be explained, according Nuruddin, due to a disturbance in a crystalline region during ball milling, which does not occur during acid hydrolysis ${ }^{19}$.

\section{(iii) Scanning electron microscopy (SEM)}

Figures 3 (a) and (b) show micrographs of CNS-chemical and CNS-mechanical samples. These depict the morphology of the external aspect following isolation methods. The images exhibit substantial differences in shape and agglomeration for the samples. The surface of CNS revealed the presence of organized regions, but it also shows amorphous regions; this means the isolations are partially effective. The nanostructures suspensions exhibit a precipitation in the CNS-mechanical sample before the analysis. It could be an indicator that the resulting nanostructures are not stable and the precipitation occurs due to the deposition of aggregates. In the CNSchemical samples, the use of sulfuric acid dissolved the amorphous region and induced the grafting of negativelycharged sulfate groups on the CNS surface. These results contribute to stabilizing the colloidal suspension by repulsive interparticle forces ${ }^{17,19,20}$.
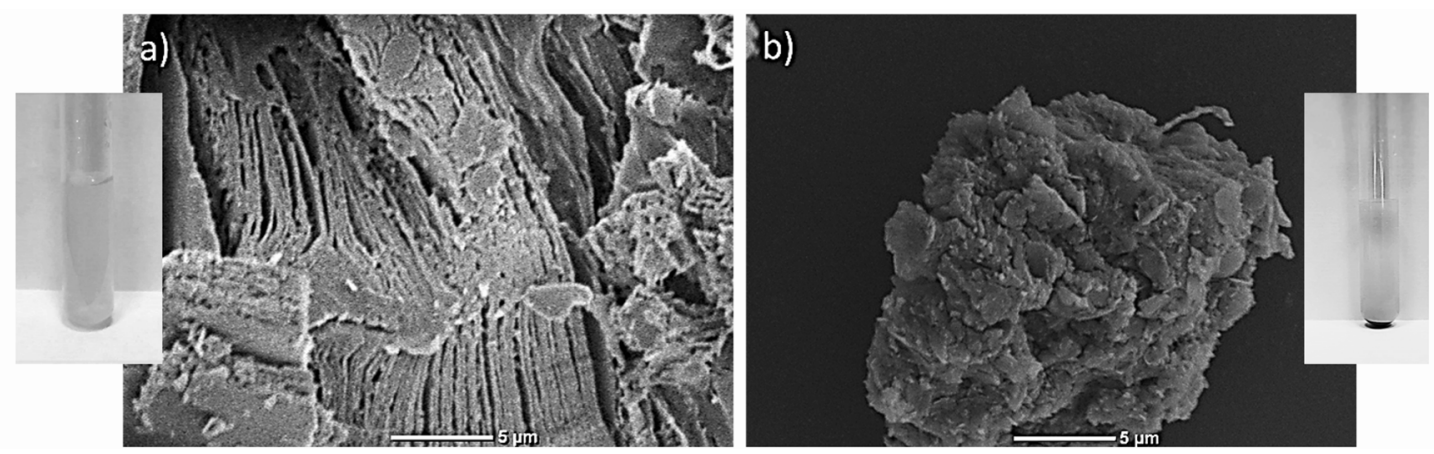

Figure 3. Scanning electron micrographs (4000x) of (a) CNS-chemical sample and (b) CNS-mechanical sample. 
(iv) Dynamic light scattering (DLS)

DLS is an effective tool to approximate particle size distribution of nano-sized materials. This analysis treats CNS as spheres moving through Brownian motion. Measuring the translational diffusion coefficient of the particles, which is converted to hydrodynamic radius $\left(\mathrm{R}_{\mathrm{H}}\right)^{21}$. Figure 4 shows the particle-size distribution of CNS-chemical and CNS-mechanical samples. It indicated the average particle size of $246 \mathrm{~nm}$ for the CNS-chemical and $281 \mathrm{~nm}$ for the CNS-mechanical. Both samples showed two peaks. For CNS-chemical shown in Figure 4(a), the major peak was recorded with average particle size of $225 \mathrm{~nm}$ ( $88 \%$ intensity) and a minor peak with average of $2 \mu \mathrm{m}(12 \%$ intensity). Chemical isolation results in a dissolution of parts of cellulose, and the nanostructures show minor sizes. For CNS-mechanical shown in Figure 4 (b), the peak was recorded with an average particle size of $281 \mathrm{~nm}$. The mechanical isolation showed higher sizes due the breakdown of weaker interactions of cellulose fiber ${ }^{6,9}$. (v) Atomic force microscopy (AFM)

Aggregation, bundling, formation of nanofibrils networks and overlapping of individual CNS make getting accurate dimension measurements difficult, even when using current image analysis techniques. For this reason, identifying the sizes of the samples is difficult ${ }^{22}$. The surface topography of the CNS-chemical and CNS-mechanical samples probed by AFM (Figure 5), showed that the two sample preparation procedures used induce different morphological features. It seems that the acid hydrolysis gives rise to elongated CNS (nanorods) $^{23}$, whereas the mechanical treatment induces the formation of nanoneedles with a mean diameter of $48 \pm 6 \mathrm{~nm}$; that is, the difference between AFM and DLS results occurs due to spherical approximation in DLS analysis. Previous studies show that nanoparticles obtained by acid hydrolysis show spherical particles and surface roughness due to highly aggregated $^{23}$. The nanorods have a mean diameter of $245 \pm 31$ $\mathrm{nm}$ and a mean length of $427 \pm 49 \mathrm{~nm}$. The average elliptic a)

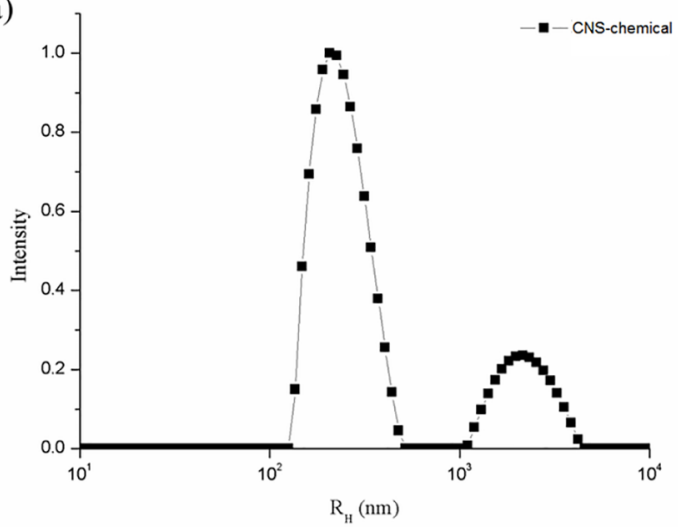

b)

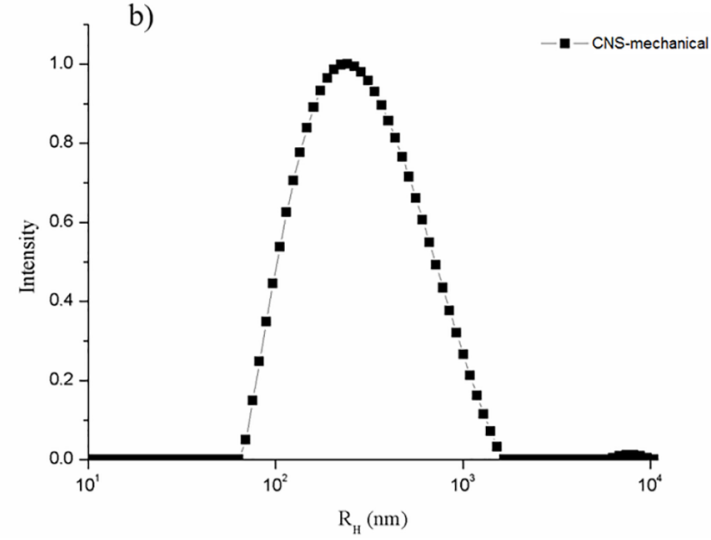

Figure 4. Scattering intensity curves as a function of the hydrodynamic radius $\left(\mathrm{R}_{\mathrm{H}}\right)$ of (a) CNS-chemical sample and (b) CNS-mechanical sample.
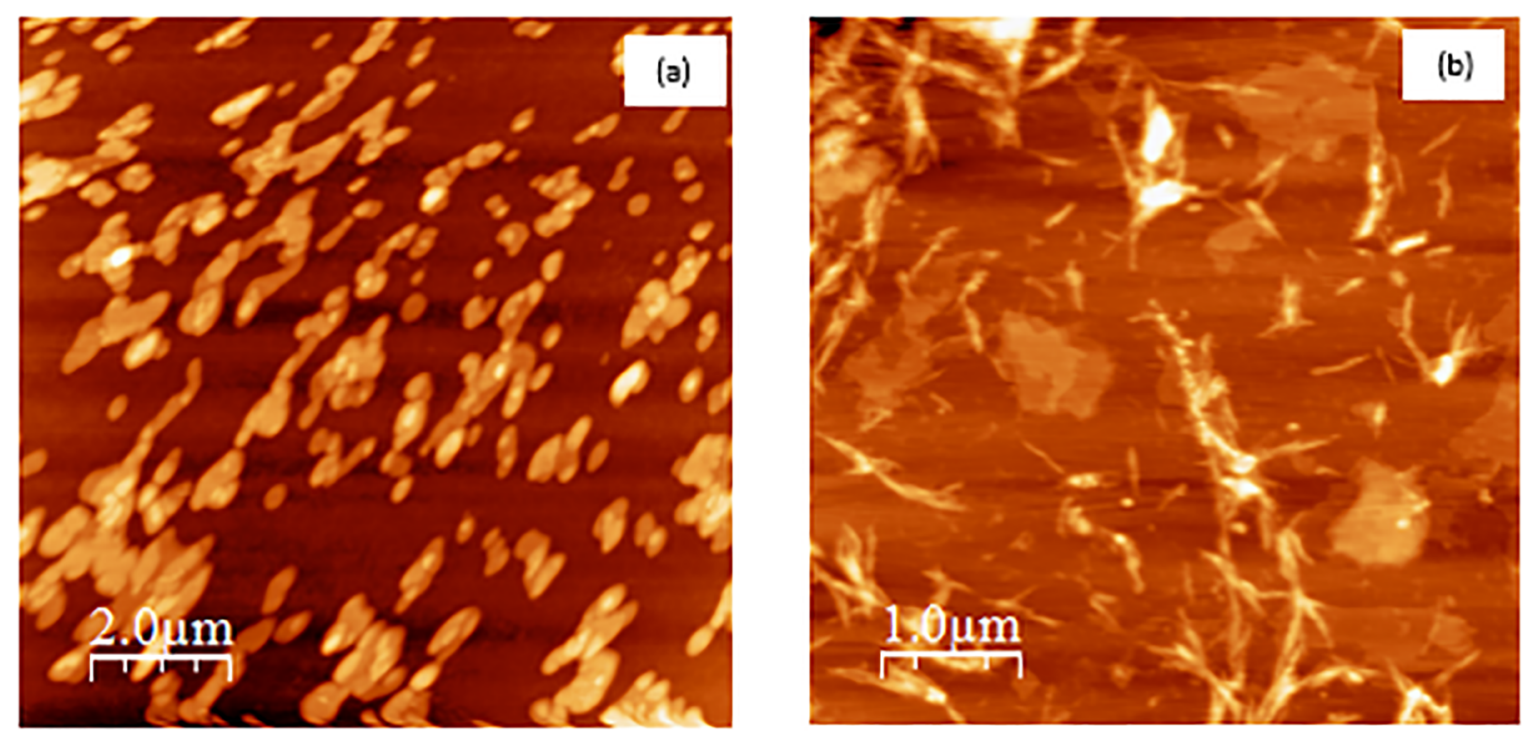

Figure 5. AFM image of (a) CNS-chemical sample and (b) CNS-mechanical sample. 

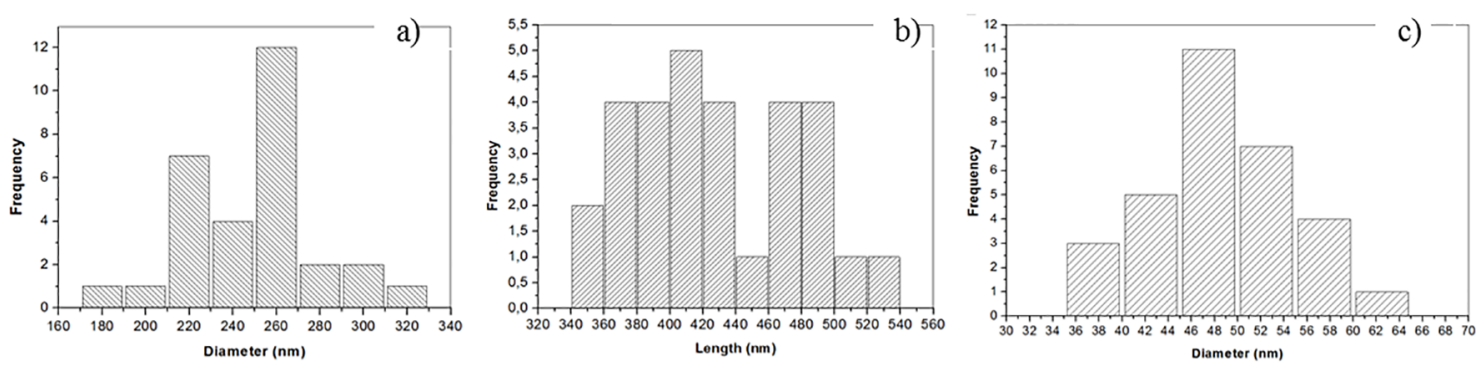

Figure 6. Histogram distribution of the CNS (a) diameter of CNS-chemical sample and (b) length of CNS-chemical; (c) diameter of CNS-mechanical sample.

ratio of the cellulose nanostructures is then of about 1.75 . Size measurements of individual CNS were based on the evaluation of AFM images and the histogram distributions of the nanorod diameters and lengths observed for CNS-chemical samples, as shown in Figures $6 a$ and $6 b$, respectively. The diameter distribution histogram of the nanoneedles obtained from the CNS-mechanical sample is shown in Figure 6c. This CNS size and morphology dependence has been frequently reported in the literature ${ }^{24,25}$.

\section{Conclusions}

Nanocellulose was isolated from paper waste via sulfuric acid hydrolysis and mechanical process to obtain distinct morphologies and surface properties. Both samples show purified cellulose, as confirmed by the FTIR results. TGA revealed lower thermal stability for CNS-mechanical samples than for CNS-chemical samples, which was probably caused by the residual cellulose amorphous region. In SEM, different morphologies were observed, with both samples displaying a tendency to agglomerate. The average CNS sizes varied with the method: $246 \mathrm{~nm}$ for CNS-chemical samples and $281 \mathrm{~nm}$ for CNS-mechanical samples. AFM analysis showed different morphologies, with cellulose nanostructures obtained by sulfuric acid hydrolysis and nanoneedles obtained by a mechanical method. Therefore, we believe that in the near future, paper industrial residues containing high cellulose content will prove to be a very promising source of low-cost, raw material for CNS production, as well as for other high value-added bio-conversion processes.

\section{Acknowledgments}

The authors thank the UFABC and CNPq. This work was supported by CNPq (grant numbers 306401/2013-4, 447180/2014-2, 830094/2008-3).

\section{References}

1. Väisänen T, Haapala A, Lappalainen R, Tomppo L. Utilization of agricultural and forest industry waste and residues in natural fiber-polymer composites: A review. Waste Management. 2016;54:62-73.
2. Thompson G, Swain J, Kay M, Forter CF. The treatment of pulp and paper mill effluent: a review. Bioresource Technology. 2001;77(3):275-286 .

3. Tuzzin G, Godinho M, Dettmer A, Zattera AJ. Nanofibrillated cellulose from tobacco industry wastes. Carbohydrate Polymers. 2016;148:69-77.

4. Xiong R, Zhang X, Tian D, Zhou Z, Lu C. Comparing microcrystalline with spherical nanocrystalline cellulose from waste cotton fabrics. Cellulose. 2012;19(4):1189-1198.

5. Mondal S. Preparation, properties and applications of nanocellulosic materials. Carbohydrate Polymers. 2017;163:301-316.

6. Abdul Khalil HPS, Davoudpour Y, Islam N, Mustapha A, Sudesh K, Dungani R, et al. Production and modification of nanofibrillated cellulose using various mechanical processes: A review. Carbohydrate Polymers. 2014;99:649-665.

7. Martínez-Lage I, Velay-Lizancos M, Vázquez-Burgo P, RivasFernandez M, Vazquez-Herrero C, Ramírez-Rodríguez A, et al. Concretes and mortars with waste paper industry: Biomass ash and dregs. Journal of Environmental Management. 2016;181:863-873.

8. Lani NS, Ngadi N, Johari A, Jusoh M. Isolation, characterization and application of nanocellulose from oil palm empty fruit bunch fiber as nanocomposites. Journal of Nanomaterials. 2014;2014:702538.

9. Oun AA, Rhim JW. Characterization of nanocelluloses isolated from Ushar (Calotropis procera) seed fiber: Effect of isolation method. Materials Letters. 2016;168:146-150.

10. Johar N, Ahmad I, Dufresne A. Extraction, preparation and characterization of cellulose fibres and nanocrystals from rice rusk. Industrial Crops and Products. 2012;37(1):93-99.

11. Jiang F, Hsieh YL. Cellulose nanocrystal isolation from tomato peels and assembled nanofibers. Carbohydrate Polymers. 2015;122:60-68.

12. Bellamy WD. Single cell proteins from cellulosic wastes. Biotechnology and Bioengineering. 1974;16(7):869-880.

13. Chandra CSJ, George N, Narayanankutty SK. Isolation and characterization of cellulose nanofibrils from arecanut husk fibre. Carbohydrate Polymers. 2016;142:158-166.

14. Chen YW, Lee HV, Juan JC, Phang SM. Production of new cellulose nanomaterial from red algae marine biomass Gelidium elegans. Carbohydrate Polymers. 2016;151:1210-1219. 
15. Fortunati E, Puglia D, Monti M, Peponi L, Santulli C, Kenny $\mathrm{JM}$, et al. Extraction of Cellulose Nanocrystals from Phormium tenax Fibres. Journal of Polymers and the Environment. 2013;21(2):319-328.

16 Sá RM, Miranda CS, José NM. Preparation and Characterization of Nanowhiskers Celulose from Fiber Arrowroot (Maranta arudinacea). Materials Research. 2015;18(Suppl. 2):225-229.

17. Abraham E, Deepa B, Pothen LA, Cintil J, Thomas S, John $\mathrm{MJ}$, et al. Environmental friendly method for the extraction of coir fibre and isolation of nanofibre. Carbohydrate Polymers. 2013;92(2):1477-1483.

18. Mariano M, Cercená R, Soldi V. Thermal characterization of cellulose nanocrystals isolated from sisal fibers using acid hydrolysis. Industrial Crops and Products. 2016;94:454-462.

19. Nuruddin M, Hosur M, Uddin MJ, Baah D, Jeelani A. A novel approach for extracting cellulose nanofibers from lignocellulosic biomass by ball milling combined with chemical treatment. Journal of Applied Polymer Science. 2016;133(9):42990.

20. Oliveira FB, Bras J, Pimenta MTB, Curvelo AAS, Belgacem MN. Production of cellulose nanocrystals from sugarcane bagasse fibers and pith. Industrial Crops and Products. 2016;93:48-57.
21. Boluk Y, Danumah C. Analysis of cellulose nanocrystal rod lengths by dynamic light scattering and electron microscopy. Journal of Nanoparticle Research. 2014;16:2174.

22. Sacui IA, Nieuwendaal RC, Burnett DJ, Stranick SJ, Jorfi M, Weder C, et al. Comparison of the Properties of Cellulose Nanocrystals and Cellulose Nanofibrils Isolated from Bacteria, Tunicate, and Wood Processed Using Acid, Enzymatic, Mechanical and Oxidative Methods. ACS Applied Materials \& Interfaces. 2014;6(9):6127-6138

23. Mohamad Haafiz MK, Eichhorn SJ, Hassan A, Jawaid M. Isolation and characterization of microcrystalline cellulose from oil palm biomass residue. Carbohydrate Polymers. 2013;93(2):628-634.

24. Wang H, Zhang X, Jiang Z, Li W, Yu Y. A comparison study on the preparation of nanocellulose fibrils from fibers and parenchymal cells in bamboo (Phyllostachys pubescens). Industrial Crops and Products. 2015;71:80-88.

25. Jiang F, Hsieh YL. Chemically and mechanically isolated nanocellulose and their self-assembled structures. Carbohydrate Polymers. 2013;95(1):32-40. 